\title{
Molecular Dynamics Simulation of Spinodal Decomposition in Three-Dimensional Binary Fluids
}

\section{Laradji, Mohamed; Toxvaerd, Søren; Mouritsen, Ole G.}

\section{Published in:}

Physical Review Letters

Link to article, DOI:

10.1103/PhysRevLett.77.2253

Publication date:

1996

Document Version

Publisher's PDF, also known as Version of record

Link back to DTU Orbit

\section{Citation (APA):}

Laradji, M., Toxvaerd, S., \& Mouritsen, O. G. (1996). Molecular Dynamics Simulation of Spinodal Decomposition in Three-Dimensional Binary Fluids. Physical Review Letters, 77(11), 2253-2256.

https://doi.org/10.1103/PhysRevLett.77.2253

\section{General rights}

Copyright and moral rights for the publications made accessible in the public portal are retained by the authors and/or other copyright owners and it is a condition of accessing publications that users recognise and abide by the legal requirements associated with these rights.

- Users may download and print one copy of any publication from the public portal for the purpose of private study or research.

- You may not further distribute the material or use it for any profit-making activity or commercial gain

- You may freely distribute the URL identifying the publication in the public portal 


\title{
Molecular Dynamics Simulation of Spinodal Decomposition in Three-Dimensional Binary Fluids
}

\author{
Mohamed Laradji, ${ }^{1}$ Søren Toxvaerd, ${ }^{2}$ and Ole G. Mouritsen ${ }^{3}$ \\ ${ }^{1}$ Department of Physics, University of Toronto, Toronto, Ontario, Canada M5S 1A7 \\ ${ }^{2}$ Department of Chemistry, H. C. Ørsted Institute, University of Copenhagen, DK-2100 Copenhagen, Denmark \\ ${ }^{3}$ Department of Physical Chemistry, The Technical University of Denmark, Building 206, DK-2800 Lyngby, Denmark
}

(Received 17 April 1996)

\begin{abstract}
Using large-scale molecular dynamics simulations of a two-component Lennard-Jones model in three dimensions, we show that the late-time dynamics of spinodal decomposition in concentrated binary fluids reaches a viscous scaling regime with a growth exponent $n=1$, in agreement with experiments and a theoretical analysis for viscous growth. [S0031-9007(96)01177-5]
\end{abstract}

PACS numbers: 64.75.+g, 05.70.Ln, 64.60.Cn, 64.70.Ja

The dynamics of phase separation in multicomponent fluids involves very rich and general phenomena and has therefore been the subject of intensive studies in recent years. The dynamics of first-order phase transitions in general, besides being of technological importance, is particularly interesting because of the emergence of one characteristic length scale $R(t)$ during the late times of the dynamics. $R(t)$ is related to the average domain size of the ordering phase and displays a simple power-law dependence with time $R(t) \sim t^{n}$, where $n$ is the growth exponent. The presence of one characteristic length scale during the late times leads to an interesting dynamical scaling behavior, as can be detected from the densityfluctuation pair-correlation function $G(\mathbf{r}, t)=g[\mathbf{r} / R(t)]$, or the structure factor $S(\mathbf{q}, t)=R(t)^{d} F(\chi)$, where $d$ is the spatial dimension and $\chi=\mathbf{q} R(t)$ is the scaled wave vector [1].

Whereas the dynamics of phase separation in alloys, with conserved order parameter, is quite well understood in terms of the Lifshitz-Slyozov theory [2] and is characterized by a growth exponent $n=1 / 3$, independent of spatial dimension, volume fraction [1], and even the number of coexisting phases [3], the dynamics in fluids is a more complicated phenomenon due to the coupling of the additional velocity field (which is absent in alloys) to the ordering field. Consequently various competing effects may appear in phase-separating fluids leading to various growth exponents depending on the strength of the coupling between the velocity field and the ordering field, on the volume fraction [4-6], on the spatial dimension, and even on the number of components [7].

There is no satisfactory theory for the phase separation dynamics in fluids. Thus our understanding of the phenomenon is achieved essentially through numerical studies and dimensional analysis of the relevant dynamical model. Using heuristic arguments, Siggia [4] was the first to propose that the growth exponent is $n=1$ in phaseseparating binary fluids with relatively comparable volume fractions of the two components. This growth regime is due to an instability of the tubular domain structure in binary fluids, leading to the transport of material from the necks to the bulges. The numerical studies of the phenomenon are mainly carried out by means of three different methods: numerical integration of the corresponding kinetic phase-field model known as model $\mathrm{H}$ [8]; latticeBoltzmann (LB) simulations [9]; and molecular dynamics (MD) simulations [10]. In contrast to the first two methods, in a molecular dynamics simulation, the hydrodynamic modes arise naturally from the microscopic interactions between the molecules subsequent to a quench into the fluid phase. There have been some concerns with regard to the validity of molecular dynamics in studying the late-time dynamics of phase separation due to the very small time scale involved. It should be noted that phase separation in simple fluids is naturally a very fast process. Therefore in order to probe the dynamics, experimentalists must perform very shallow quenches using the advantage of the increased time scale due to critical slowing down. In contrast, quenches are very deep in a typical molecular dynamics simulation.

The numerical integration of model $\mathrm{H}$ leads to an asymptotic growth exponent $n=1$, in agreement with Siggia's prediction. LB simulations also find the same result [9]. However, a recent MD simulation on the two-component Lennard-Jones potential by Ma et al. [10] suggests a growth regime with an exponent very close to $2 / 3$. As we will see later, such an exponent is due to inertial effects and can be calculated from dimensional analysis. A more recent model $\mathrm{H}$ simulation by Lookman et al. [11] finds that by decreasing the shear viscosity of the fluid, a growth exponent of $n=2 / 3$ can be observed. We are therefore faced with the problem that whereas numerical simulation calculations in the case of phase separation in alloys agree with the theoretical predictions, numerical simulations which are expected to most faithfully describe the true dynamics, i.e., molecular dynamics simulations, are not in agreement with theoretical predictions in the case of phase separation in binary fluids. In order to elucidate this apparent discrepancy between the previous numerical studies and the MD simulations of Ma et al., we have carried out a large-scale and systematic molecular dynamics simulation 
of the two-component Lennard-Jones model and found results which disagree with the MD simulation of Ma et al. but are fully consistent with experiments and previous model $\mathrm{H}$ and lattice-Boltzmann simulations. It is worth noting that the present study is the first large-scale MD simulation on three-dimensional binary fluids in which the viscous regime is observed.

In our simulation model, we consider $N$ monoatomic molecules interacting through the following twocomponent Lennard-Jones potential:

$$
\begin{aligned}
U_{\alpha_{i}, \alpha_{j}}\left(\mathbf{r}_{i j}\right)=4 \epsilon & \left\{\left[\left(\frac{\sigma}{r_{i j}}\right)^{12}-\left(\frac{\sigma}{r_{i j}}\right)^{6}\right]\right. \\
& \left.-\left[\left(\frac{\sigma}{r_{\alpha_{i} \alpha_{j}}^{c}}\right)^{12}-\left(\frac{\sigma}{r_{\alpha_{i} \alpha_{j}}^{c}}\right)^{6}\right]\right\} \\
& \times \theta\left(r_{\alpha_{i}, \alpha_{j}}^{c}-r_{i j}\right),
\end{aligned}
$$

with $\alpha_{i}=1$ if $i$ is an $A$ molecule, and $\alpha_{j}=2$ if $i$ is a $B$ molecule. In Eq. (1), $r_{i j}$ is the distance separating the $i$ th molecule from the $j$ th molecule, and $r_{\alpha_{i} \alpha_{j}}^{c}$ is a cutoff distance which is equal to $2.5 \sigma$ for $\alpha_{i}=\alpha_{j}$ and $2^{1 / 6} \sigma$ for $\alpha_{i} \neq \alpha_{j} . \quad \theta(x)$ is the standard Heaviside function. The phase diagram of this model, which has recently been calculated by means of mean field theory and Monte Carlo simulation, has a consolute point at $T_{c} \sim(4.7 \pm 0.2) \epsilon$ for a fluid density of $\rho=0.8 \sigma^{-3}$ [12]. We have performed critical quenches at temperatures $k_{B} T=2,3,3.5,3.75$, and $4 \epsilon$ as well as off-critical quenches at $k_{B} T=2 \epsilon$. Notice that we have not made quenches to very low temperatures in order to avoid the solid-gas coexistence region. The temperature is controlled by a Nosé-Hoover thermostat [13], and the Hamilton equations are integrated using the leap-frog algorithm with a time step of $\Delta t=$ $0.005 \tau$ where the time scale is $\tau=\sqrt{\mu \sigma^{2} / \epsilon}, \mu$ being the molecular mass. In all of our simulations, the total number of molecules is $N=343000$, an order of magnitude larger than the largest system size considered by Ma et al. [10]. Our simulations were performed on an IBM SP2 parallel machine using 12 processors. Furthermore, a statistical average is performed for each quench; 16 runs for $k_{B} T=2 \epsilon$ and 4 runs for all other quenches.

We have calculated the correlation function $G(\mathbf{r}, t)=$ $\langle\phi(\mathbf{r}, t) \phi(0, t)\rangle$, where $\phi(\mathbf{r}, t)=\left[\rho_{A}(\mathbf{r}, t)-\rho_{B}(\mathbf{r}, t)\right] / \rho$ is the order parameter and $\rho_{A}$ and $\rho_{B}$ are the local densities of the two components. We have also calculated the structure factor $S(\mathbf{q}, t)=\left\langle|\tilde{\phi}(\mathbf{q}, t)|^{2}\right\rangle / V$, where $\tilde{\phi}(\mathbf{q})$ is the Fourier-mode of the order parameter and $V$ is the system volume. Both the structure factor and the correlation function are then spherically averaged. The average domain size is then defined as the first zero of the correlation function $R_{G}(t)$ and as the $n$th moment of the structure factor $R_{n}(t)=2 \pi\left[\int d q S(q, t) / \int d q q^{n} S(q, t)\right]^{1 / n}$.

The time evolution of the pair-correlation function is shown in the inset of Fig. 1 for a quench at $k_{B} T=2 \epsilon$. The presence of the decaying oscillations in $G(r, t)$ in- dicates the occurrence of phase-separated domains which are correlated within short distances, due to the conservation of the ordering field. The first zero of the correlation function increases with time implying a coarsening of the domain structure. We have verified that the system has reached a dynamical scaling regime by observing the scaling of the correlation function, shown in Fig. 1, for times larger than about $t=80 \tau$. Good scaling is also observed in the structure factor (not shown). The presence of a unique length scale in the system at late times implies that the width of the interfaces become vanishingly small compared to the domain size. As a result, the structure factor should scale as $q^{-(d+1)}$ for large $q$, which is known as Porod's law and is usually observed in phase-separating systems at late times. Indeed we found that the structure factor is consistent with Porod's law, implying that the phase separation process in our simulations is well within a dynamical scaling regime.

Now that we are confident that the systems we are dealing with in our simulations are safely within a scaling regime, we turn to the discussion of the nature of the growth law. In Fig. 2, the time dependence of the average domain size, as calculated from the various definitions, is shown. Notice the linear dependence of $R(t)$ at late times indicating that the growth regime should be viscous, in agreement with Siggia's prediction [4]. However, when plotting the data in a double-logarithmic plot, we find that the late-time growth exponent is more consistent with $2 / 3$, possibly indicating that the observed growth regime is inertial, as suggested by the MD simulation of $\mathrm{Ma}$ et al. [10]. It should be pointed out, however, that the growth law $R(t)=R(0)+$ at investigated over a finite time range may show a growth exponent which is smaller than one due to a nonnegligible value of $R(0)$ and possible other nonalgebraic dependences.

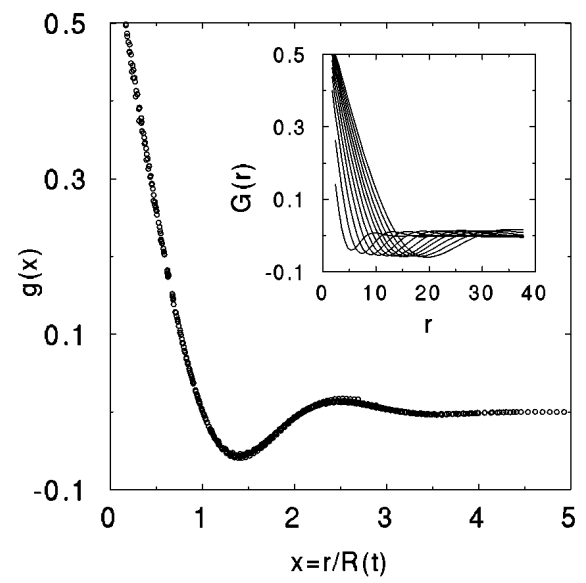

FIG. 1. Scaled pair-correlation function $g(x)$ versus the scaled distance $x=r / R(t)$ for a quench at $k_{B} T=2 \epsilon$. The data shown range from $t=80 \tau$ to $220 \tau$. The inset shows the time evolution of the correlation function from $t=20 \tau$ to $220 \tau$ in steps of $20 \tau$. 


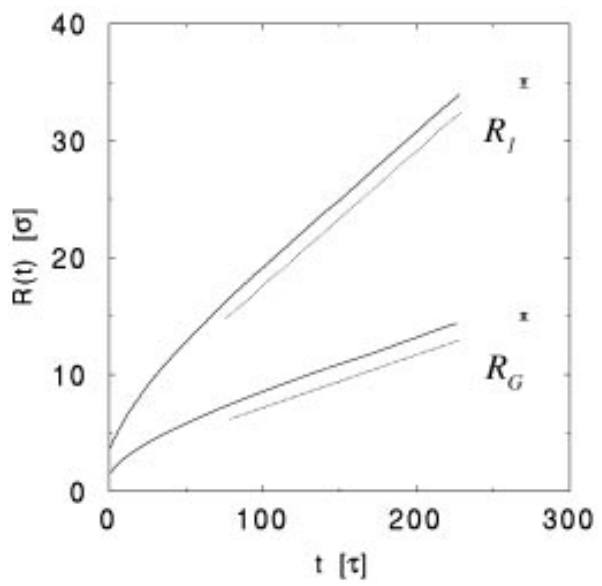

FIG. 2. The average domain size as a function of time for a quench at $k_{B} T=2 \epsilon . \quad R_{G}(t)$ is the first zero of the paircorrelation function, and $R_{1}(t)$ is calculated from the first moment of the structure factor. The two dots indicate the typical size of the error bars in the numerical data, and the two dotted lines are straight lines.

In order to determine the true asymptotic growth law, we have to consider the relevant dynamical model and analyze our results in light of its implications. The dynamics of phase separation in fluids can be described by the so-called model $\mathrm{H}$ [14], corresponding to a generalized Cahn-Hilliard equation coupled to the NavierStokes equation. The appropriate dynamical equations can then be written as follows:

$$
\begin{aligned}
\frac{\partial \phi(\mathbf{r}, t)}{\partial t}+\mathbf{v} \cdot \nabla \phi(\mathbf{r}, t)= & M \nabla^{2} \frac{\delta \mathcal{F}\{\phi\}}{\delta \phi(\mathbf{r}, t)} \\
\rho\left[\frac{\partial \mathbf{v}(\mathbf{r}, t)}{\partial t}+[\mathbf{v}(\mathbf{r}, t) \cdot \nabla] \mathbf{v}(\mathbf{r}, t)\right]= & \eta \nabla^{2} \mathbf{v}(\mathbf{r}, t) \\
& -\nabla p(\mathbf{r}, t)-\phi(\mathbf{r}, t) \\
& \times \nabla \frac{\delta \mathcal{F}\{\phi\}}{\delta \phi(\mathbf{r}, t)}
\end{aligned}
$$

where $\phi(\mathbf{r}, t), \mathbf{v}(\mathbf{r}, t)$, and $p(\mathbf{r}, t)$ are the local order parameter, the velocity field, and the pressure field, respectively. The constants $M, \rho$, and $\eta$ correspond to the order parameter mobility, the fluid density, and the shear viscosity, respectively. $\mathcal{F}$ is the usual $\phi^{4}$ free energy functional [1]. The difference between Eq. (2) and the usual Cahn-Hilliard equation is the presence of the second term on the left-hand side, which accounts for the transport of the order parameter by the velocity field. Equation (3) is different from the usual Navier-Stokes equation by the presence of the additional force acting on the fluid due to gradients in the chemical potential.

The set of equations (2) and (3) is very difficult to solve, but one can obtain various growth regimes by means of simple dimensional analysis. Here we will limit ourselves to three dimensions. At relatively early times, but late enough so that the domains are well defined and much larger than the interfacial width, the velocity field is decoupled from the order parameter leading to the usual Lifshitz-Slyozov growth law usually observed in alloys $R(t) \sim(M \gamma t)^{1 / 3}$, where $\gamma$ is the interfacial tension [2]. This regime will be referred to as the diffusive regime. At later times, the coupling between $\phi$ and $\mathbf{v}$ cannot be neglected but the inertial term in Eq. (3), can be neglected so that $\mathbf{v}$ becomes slaved by $\phi$. One thus obtains the following growth law, $R(t) \sim(\gamma t / \eta)$, which will be associated with a viscous regime and has been predicted by Siggia [4] as a consequence of a necking-down instability of the tubular (interconnected) domain structure due to the transport of material from the necks to the bulges. This regime has been observed in several simple binary fluids and binary homopolymer blends $[15,16]$ as well as in numerical simulations [8,9]. At even later times, the inertial term in the Navier-Stokes equation can no longer be neglected, and one finds the growth law of the inertial regime $R(t) \sim(\gamma / \rho)^{1 / 3} t^{2 / 3}$ [5]. The two last regimes can be observed only for interconnected domain structures. For dilute binary solutions, the domains are dropletlike, and the domain growth is essentially due to their coalescence leading to a growth law $R(t) \sim t^{1 / 3}$, but with a prefactor which is larger than that in the diffusive regime. The inertial regime has not been observed experimentally, but it has been observed in several numerical simulations in two dimensions $[9,17,18]$. Introducing two time scales, $t_{v}=(\gamma / \eta) t$ and $t_{i}=(\gamma / \rho)^{1 / 3} t^{2 / 3}$ for the viscous regime and the inertial regime, respectively, the $t_{v}$ dependence ( $t_{i}$ dependence) of $R(t)$ during the viscous (inertial) regime must be linear and independent of the quench depth, except maybe for interference with $R(0)$.

We have therefore calculated, by molecular dynamics simulations, the interfacial tension $\gamma$ and the shear viscosity $\eta$ for the various quench temperatures considered in the present study. We obtain a shear viscosity which is practically independent of temperature and equal to $\eta=1.65$. However, the interfacial tension is found to decrease with temperature, almost linearly from $(1.85 \pm 0.07) \epsilon / \sigma^{2}$ for $k_{B} T=2 \epsilon$ to $(0.39 \pm 0.13) \epsilon / \sigma^{2}$ for $k_{B} T=4 \epsilon$, since the present model belongs to the Ising universality class in $d=3$. In Fig. 3(a), $R(t)$ is plotted versus $t_{v}=(\gamma t / \eta)$, and in Fig. 3(b), $R(t)$ is plotted versus $t_{i}=(\gamma / \rho)^{1 / 3} t^{2 / 3}$ for all quench temperatures. Although the data are almost linear with $t_{i}$ for all temperatures, the slope of $R(t)$ versus $t_{i}$ depends strongly on $T$, whereas the slope of $R(t)$ versus $t_{v}$ is independent of temperature. This, therefore, strongly indicates that the growth regime found in this system cannot be inertial, in contrast to the prediction by Ma et al. [10], but in agreement with the other numerical studies and experiments. In our simulations, dynamical scaling is observed starting from $t=80 \tau$ at the lowest quench temperatures. At higher temperatures, the scaling regime is delayed to later times. This is to be contrasted to the study of Ma et al., in which it was found that the scaling regime starts as early as $20 \tau$ [10]. Of course, the fact that we did not 

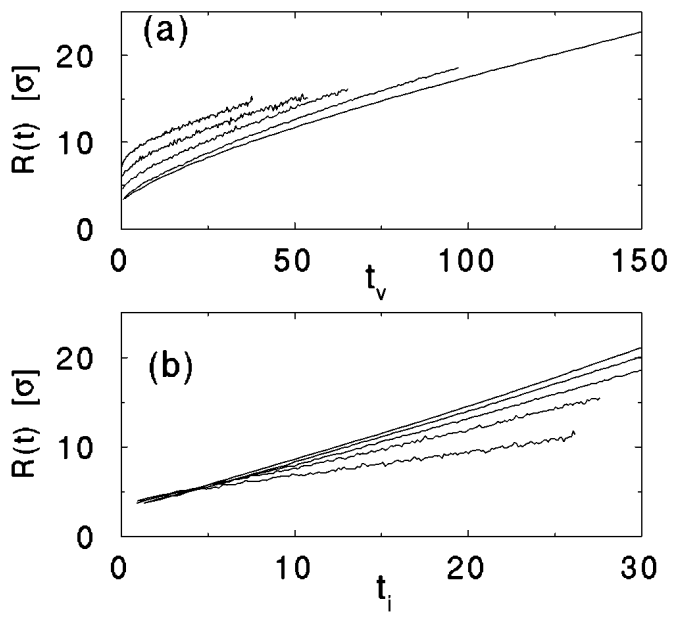

FIG. 3. The average domain size as a function of $t_{v}=$ $(\gamma / \eta) t$. Data lines from bottom to top correspond to $k_{B} T=$ $2 \epsilon, 3 \epsilon, 3.5 \epsilon, 3.75 \epsilon$, and $4 \epsilon$, respectively. For the sake of clarity, data have been shifted vertically upward. (b) The average domain size as a function of $t_{i}=(\gamma / \rho)^{1 / 3} t^{2 / 3}$. Data from top to bottom correspond to $k_{B} T=2 \epsilon, 3 \epsilon, 3.5 \epsilon, 3.75 \epsilon$, and $4 \epsilon$, respectively.

observe an inertial regime does not disprove the presence of this regime at even later times, as predicted by the scaling analysis. The inertial regime has been observed in previous numerical studies in two dimensions [11,17], and in a recent model $\mathrm{H}$ simulation in three dimensions [11]. In order to detect such a regime, we must simulate much larger systems.

Another reason, making us even more confident that the dynamical regime found in the present study is viscous, is the value of the prefactor of the growth law in terms of $t_{v}$. Siggia predicted that this prefactor is 0.6 , whereas San Miguel, Grant, and Gunton [6] find that it should be 0.25 from a linear stability analysis of the tubular structure. However, a detailed experimental study by Guenoun et al. finds that the prefactor is $0.138 \pm 0.006$ [16]. In our simulation we find the prefactor to be $0.11 \pm 0.01$, which is very close to the experimental value of Guenoun et al., but disagrees with the two theoretical predictions which, however, are quite crude in nature. The difference between the value of our prefactor and that of Guenoun et al. might be due to the finite size of our systems, leading to a cutoff of the long-range hydrodynamic modes. Indeed, one expects that this prefactor decreases linearly with $1 / L$ from its thermodynamics-limit value [19]. Moreover, we found that the prefactor of $t_{v}$ is independent of volume fraction for quenches at volume fractions around 0.5. However, for volume fractions smaller than about 0.3, we found a growth exponent consistent with $1 / 3$. We note that recently Nikolayev et al. have predicted that a sharp transition from the viscous growth to coalescence-dominated growth occurs at a volume fraction around 0.3 [20].

In conclusion, we have performed a large-scale systematic molecular dynamics study of the phase separa- tion dynamics in binary fluids in three dimensions which faithfully accounts for hydrodynamic modes. During late times, the system reaches a dynamical scaling regime during which the average domain size grows linearly with time in agreement with Siggia's prediction, previous numerical integration of model $\mathrm{H}$, and lattice-Boltzmann simulations. The discrepancy with a previous molecular dynamics study has been clarified.

The authors would like to thank R.C. Desai and P. Padilla for useful discussions. This work was supported by the Danish Natural Science Research Council, the Danish Technical Research Council, and by the Natural Sciences and Engineering Research Council of Canada.

[1] J. D. Gunton, M. San Miguel, and P.S. Sahni, in Phase Transitions and Critical Phenomena, edited by C. Domb and J.L. Lebowitz (Academic Press, New York, 1983), Vol. 8, p. 265, and references therein.

[2] I. L. Lifshitz and V. V. Slyozov, J. Phys. Chem. Solids 19, 35 (1962).

[3] C. Jeppesen and O. G. Mouritsen, Phys. Rev. B 47, 14724 (1993).

[4] E. D. Siggia, Phys. Rev. A 20, 595 (1979).

[5] H. Furukawa, Adv. Phys. 34, 703 (1985).

[6] M. San Miguel, M. Grant, and J. D. Gunton, Phys. Rev. A 31, 1001 (1985).

[7] M. Laradji, O. G. Mouritsen, and S. Toxvaerd, Europhys. Lett. 28, 157 (1994); Phys. Rev. E 53, 3673 (1996).

[8] T. Koga and K. Kawasaki, Phys. Rev. A 44, R817 (1991); S. Puri and B. Dünweg, Phys. Rev. A 45, R6977 (1992); O. T. Valls and J. E. Farrell, Phys. Rev. E 47, R36 (1993); A. Shinozaki and Y. Oono, Phys. Rev. E 48, 2622 (1993).

[9] J. F. Alexander, S. Chen, and D. W. Grunau, Phys. Rev. B 48, 634 (1993); S. Chen and T. Lookman, J. Stat. Phys. 81, 223 (1995).

[10] W.-J. Ma et al., Phys. Rev. A 45, R5347 (1992).

[11] T. Lookman, Y. Wu, F. J. Alexander, and S. Chen, Phys. Rev. E 53, 5513 (1996).

[12] S. Toxvaerd and K. Velasco, Mol. Phys. 86, 845 (1995).

[13] S. Nosé, Mol. Phys. 52, 255 (1984); W. G. Hoover, Phys. Rev. A 31, 1695 (1985).

[14] B. I. Hohenberg and P. C. Halperin, Rev. Mod. Phys. 49, 435 (1977).

[15] N.-C. Wong and C.M. Knobler, Phys. Rev. A 24, 3205 (1981); F. S. Bates and P. Wiltzius, J. Chem. Phys. 91, 3258 (1989); T. Izumitani, M. Takenaka, and T. Hashimoto, J. Chem. Phys. 92, 3213 (1990).

[16] P. Guenoun, R. Gastaud, F. Perrot, and D. Beysens, Phys. Rev. A 36, 4876 (1987).

[17] E. Velasco and T. Toxvaerd, Phys. Rev. Lett. 71, 388 (1993).

[18] Y. Wu, F. J. Alexander, T. Lookman, and S. Chen, Phys. Rev. Lett. 74, 3852 (1995).

[19] B. Dünweg and K. Kremer, J. Chem. Phys. 99, 6983 (1993).

[20] V.S. Nikolayev, D. Beysens, and P. Guenoun, Phys. Rev. Lett. 76, 3144 (1996). 